\title{
Effect of Sputtering Power on Nano-mechanical Properties of SiCO Film
}

\author{
Chun-Yang LIU ${ }^{1, a}$, Ning-Bo LIAO ${ }^{1, b, *}$, Yao YANG ${ }^{1, c}$, Jie-Lin ZHANG ${ }^{1, d}$ \\ ${ }^{1}$ School of Mechanical and Electrical Engineering, Wenzhou University, Wenzhou Zhejiang 325000 , \\ China \\ a992934699@qq.com, 'Inb55@163.com, '863708649@qq.com, ${ }^{\mathrm{d}} 757477351 @ q q . c o m$ \\ ${ }^{*}$ Corresponding author
}

Keywords: SiCO, Magnetron Sputtering, Scratch Test, Nano-indentation.

\begin{abstract}
In this study, unbalanced magnetron sputtering approach was used to prepare SiC film by using high-purity Si target. SiCO films were synthesized by reactive radio-frequency sputtering from a SiC target with oxygen gas. Nano-indentation and scratch test were used to study the mechanical properties of SiCO film at different sputtering power. According to the experimental results, optimums of Young's modulus, surface roughness and adhesion strength present at an optimal sputtering power.
\end{abstract}

\section{Introduction}

Silicon carbide is the third generation of wide band gap semiconductor material with low density, high thermal conductivity, low thermal expansion coefficient, high hardness and excellent performance, the reference [1-2]. In addition, there are excellent optical properties of SiC thin film is a kind of optical film with more research value, but the $\mathrm{SiC}$ thin film in the visible light region has a certain absorption effect [3], and silicon oxide ( $\mathrm{SiOx}$ ) has high optical transmittance, and has the properties of silicon carbide films with similar, the protective film can be used as optical components [4-5]. SiCO film is a ternary glassy compound materials, at the same time with silicon carbide films and silicon oxide thin films [6-7] many excellent characteristics, such as good thermal stability, bandwidth, refractive index, high hardness, high thermal conductivity, which is a novel optical film has potential application value.

Similar to other compound thin film, silicon oxycarbide films belong to metastable materials, have a great relationship with the performance, film composition and bonding state and the preparation method, and can adjust the performance of these films by controlling process parameters. Preparation method of silicon oxycarbide thin film are the most commonly used plasma chemical vapor deposition (PECVD) [8-10] RF magnetron sputtering and ion beam synthesis [11] .

In this experiment, we used magnetron sputtering method, and SiCO thin films were prepared under different sputtering power. Then, detecting the nanomechanical properties by using the scrath test method, nano indentation test method, in order to test the influence of different sputtering power on the mechanical properties of SiCO thin films.

\section{Experimental Procedure}

In this study, the utility of domestic JSD450-III high vacuum magnetron sputtering machine, fabrication of transparent SiCO films by DC sputtering. The SiC target with purity of $99.99 \%$, was used as the substrate of monocrystalline silicon wafer. The first pre cleaning of silicon wafer, and then dried in a vacuum drying box. A sputtering target substrate distance of $6 \mathrm{~cm}$, sputtering in argon environment, during sputtering process, the substrate temperature is $400 \mathrm{DEG} \mathrm{C}$, the sputtering pressure is $0.3 \mathrm{~Pa}$, the base substrate to maintain uniform rotation, each experimental diffraction time is $120 \mathrm{~min}$. The sputtering power as design variables, $150 \mathrm{~W}, 200 \mathrm{~W}, 300 \mathrm{~W}$.

In order to ensure the reliability of the experiment, the elimination of interference factors, experimental materials and experimental equipment prior to pre cleaning. Silicon substrate cleaning 
cleaning method using RCA traditional semiconductor technology [12]. RCA cleaning fluid in twentieth Century 60's, RCA engineers Warner Kern development, is the cleaning process for the two step, to remove silicon surface organic and inorganic residues. A 1 standard cleaning liquid (RCA-1) is composed of mixed solution of deionized water, hydrogen peroxide and ammonia, can remove organic residues; A 2 standard cleaning liquid (RCA-2) is composed of deionized water, hydrogen peroxide and hydrochloric acid, can remove residual alkali metal ions, metal hydroxide and complex.

When the first cleaning with RCA-1 cleaning liquid ultrasonic cleaning for 5 minutes, then washed several times with deionized water, and then RCA-2 cleaning liquid ultrasonic cleaning for 5 minutes with deionized water washed several times. In the film before, but also for the ion beam sputtering cleaning of the substrate under high vacuum conditions. The primary function of cleaning is to remove the impurity particles deposited on the surface of the substrate, the substrate surface completely exposed atoms true. Ion bombardment can activate the substrate surface atoms, improve the polarization of substrate surface atoms, thus shortening between the deposited atoms and substrate atoms on the surface of the distance, improve two kinds of inter atomic binding energy, enhance the adhesion of the film to the substrate. At the same time, we will at the same time each experiment experimental samples prepared with three pieces of the same parameters, the late performance of different samples were measured and averaged, in order to ensure the reliability of the experimental results.

\section{Results and Discussions}

From Fig. 1, we can see when the sputtering power is $300 \mathrm{w}$, the surface roughness of the experimental film is minimal, and with the increase of sputtering power, the surface roughness of the experimental films increased first and then decreased. This is because when the power is small, With the increase of sputtering power, make the most of the sputtered particles with high energy, these particles will accelerate the impact to the surface of the films, make defects in the impact point to increase the surface roughness. While power continues to increase, from the target sputtering of Si and $\mathrm{C}$ particles increase, so as to make the film deposition rate increase, to improve the density of thin film, reduce the defect density in the thin film to reduce the surface roughness of the film.

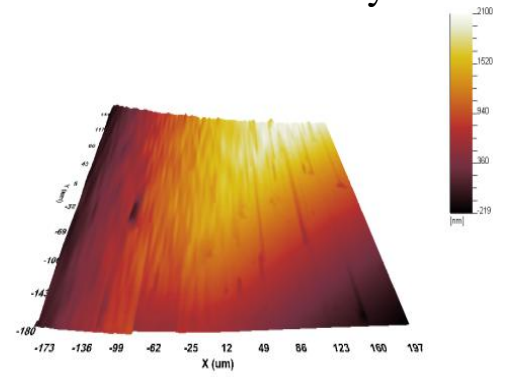

(a) $150 \mathrm{~W}$

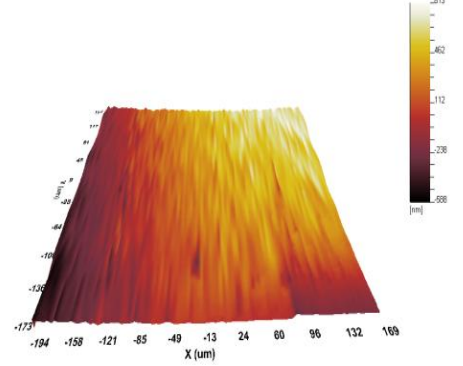

(b) $200 \mathrm{~W}$

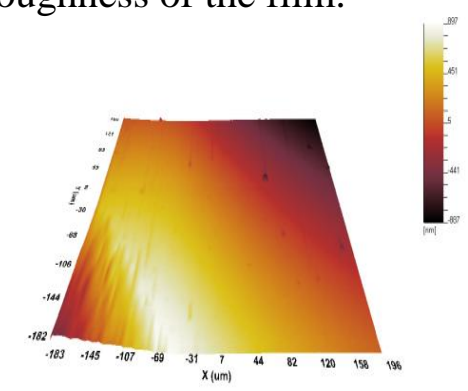

(c) $300 \mathrm{~W}$

Fig. 1 Under different sputtering power, 3 D surface topography of the thin film

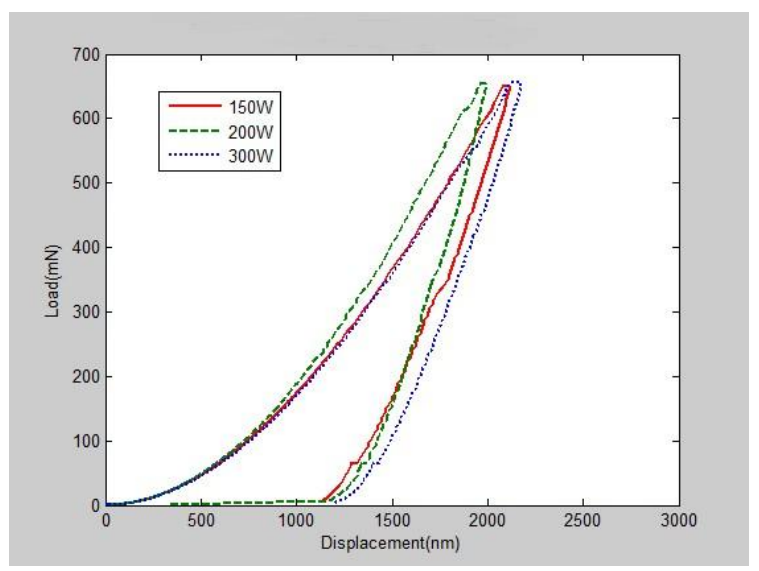

Fig. 2 The load displacement curve under different sputtering power 
Nano-indentation test is widely used to evaluate the mechanical performance of nano-materials and micro-structures. Fig. 2 shows the displacement-load curves for the samples. All the samples has obvious horizontal displacement after unloading, this show that all experimental samples have undergone plastic deformation. According to the image we can clearly observe when the load is same, the deformation of experimental sample which Sputtering power is $200 \mathrm{~W}$ is the smallest, while the deformation of experimental sample which Sputtering power is $300 \mathrm{~W}$ is the largest.

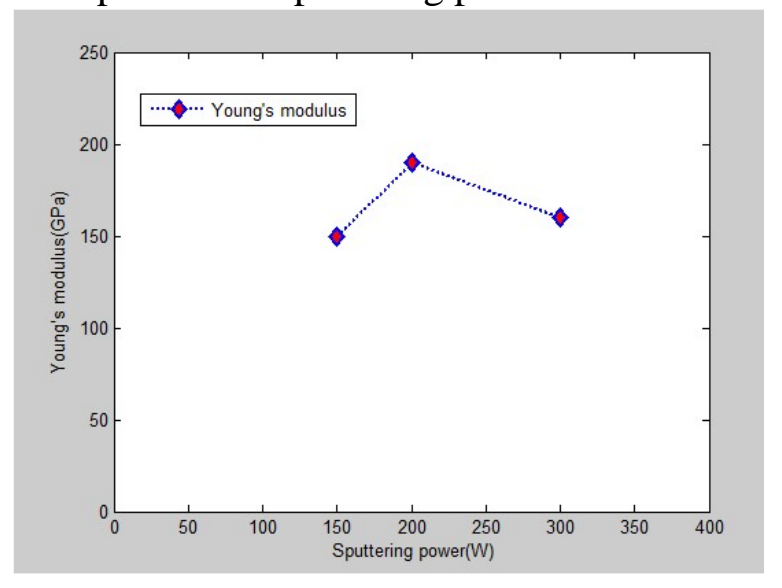

Fig. 3 The young's modulus under different sputtering power

As shown in Fig. 3, the young's modulus of experimental samples which sputtering power is $200 \mathrm{~W}$ is the largest and the young's modulus of experimental samples which sputtering power is $300 \mathrm{~W}$ is the smallest, thus we can draw a conclusion as the experimental sputtering power $200 \mathrm{~W}$ thin film resistance deformation ability was the strongest, this is due to the influence of sputtering power key in the film is not big, but for the absorption of various key strength has great influence, the greater the sputtering power, the stronger the intensity of absorption of various keys, this has a great relationship with influence of sputtering power on film thickness. Sputtering power increase, making the film thickness increases, increased levels of various key, to the number of the corresponding wave absorption enhancement, the content of the $\mathrm{C}-\mathrm{O}$ bond formation is also higher, so the modulus of elasticity is large. And the sputtering happens when power is too large, it will cause damage to membrane thus resulting in a decline in the mechanical properties of the film itself.

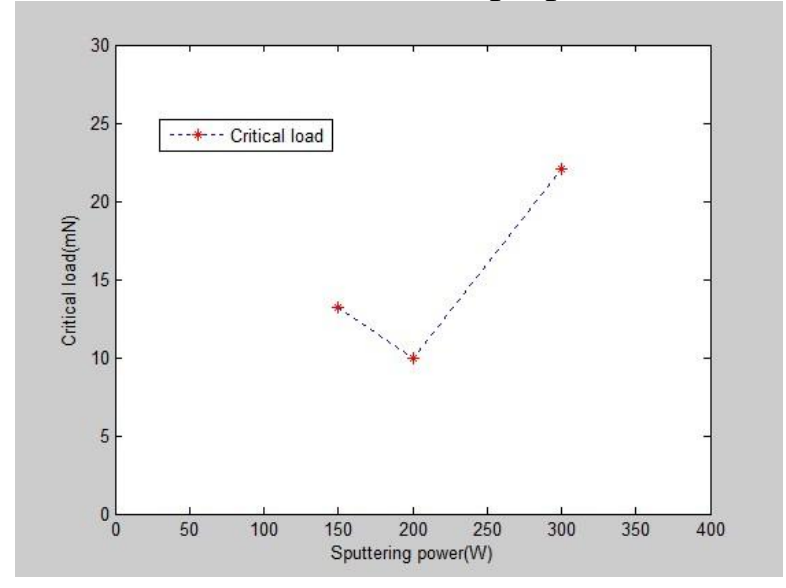

Fig. 4 The critical load under different sputtering power

Here we use acoustic emission detection method to test the binding force between the film and the substrate. The critical load values of measured membrane rupture occurs for the first time is the binding force between film and substrate material value. As we can see from fig. 4 sputtering power is $300 \mathrm{w}$ experiment the critical load of the sample is the largest, and the sputtering power is $200 \mathrm{w}$ experimental sample critical load is small. Therefore, we can draw the conclusion: with the increase of temperature, the surface of the film and the adhesive strength increased first and then decreased. 
This is because with the increase of sputtering power, the surface roughness of the films will be affected accordingly, when the surface roughness of the films decreases, the gap between the film and substrate is small, uniform film adhesion, so the film / substrate binding force is large. While the surface roughness of the films is larger, increasing the gap between the film and substrate. which leads to the decrease of the bonding strength with the matrix material, thus the performance of the film / substrate surface binding force decrease.

\section{Conclusion}

This experiment is SiCO films were prepared by magnetron sputtering, and then through the scratch test and nano-indentation test method to test out the influence of different sputtering power on the nano mechanical properties of SiCO thin films. The conclusion will be applied to the actual production and life, improve product performance, expand the actual application range of SiCO thin films. In terms of elastic modulus of the film itself, with the increase of sputtering power its performance after rising downward trend; but with the increasing of temperature, the surface roughness of the films increased first and then decreased, and decreased the membrane/adhesion is also a corresponding rise after. This is because with the increase of sputtering power, increase the content of various keys, the corresponding wave absorption enhancement so that the performance of the films improved, and too much power will cause the performance of anti sputtering thin film effect.

\section{Acknowledgement}

The authors would like to acknowledge the support of the National Natural Science Foundation of China (51202164), Qianjiang Talent Project of Zhejiang Province (2013R10068), Postdoctoral Science Foundation of China (2012M521006), China Postdoctoral Science Special Foundation (2014T70476), Research project of Educational Commission of Zhejiang Province (Y201223855), Innovative Research Team Program of Wenzhou City (C20120002-06).

\section{References}

[1] C.L. Harris. Properties of Silicon Carbide. London: INSPEC, IEE , 1995.

[2] Saddow Stephen E, Agarwal Anant. Adv. Silicon Carbide Proc. Applications. New York : Artech House, Inc, 2001.

[3] P. Zhang, C.T. Luo, X. Chen, Res. Pro. Pre method and optical properties of silicon carbide films. vacuum and low temperature, 2009, 15 (4) 193-198.

[4] H. Omanda, T. Brousse, C. Marhic, et al. Improvement of the Thermal Stability of $\mathrm{LiNi}_{0.8} \mathrm{Co}_{0.2} \mathrm{O}_{2}$ Cathode by a SiOx Protective Coating. J. Electrochem. Soc. 2004, 151 (6) 922-929.

[5] C. $\mathrm{Xu}, \mathrm{H} . \mathrm{C}$. Dong, H.J Ma, et al. Influences of $\mathrm{SiO}_{2}$ protective layers and annealing on the laser-induced damage threshold of Ta205 films. Chin. Opt. Lette, 2008, 6 (3) 228-230.

[6] C. Li, S. Chen, D.S. Wang, P. Zhang. Study on the optical properties of silicon oxycarbide thin film. J. Vac. Sci. Technol. 2012, (08) 478-483.

[7] Z.Q. Chen, H.P. Xie, L. Zhang, L.P. Guo, H.Q. Chen. Study on the structure and properties of SiCO system. J. SCNU. 2001, 6 (2) 144-154.

[8] Y.H. Wang, M.R. Moitreyee, R. Kumar, et al. Acomparative study of low dielectric constant barrier layer, etch stop and hardmask films of hydrogenated amorphous $\mathrm{Si}-(\mathrm{C}, 0, \mathrm{~N})$. Thin Solid Films. 460 (2004) 211-216.

[9] R.J. Martin-Palma, R. Gago, V.Torres-Costa, et al. Optical and compositional analysis of functional SiOxCy: H coatings on polymers. Thin Solid Films, 2006, (515) 2493-2496 
[10] V.Ryan Joseph, C.G.Pantan. Synthesis and characterization of inorganic silicon oxycarbide glass thin films by reactive Rf-magnetron sputtering. J. Vac. Sci. Technol, 2007, 25 (1) 153-159.

[11]S.L. Shevchuk, Yu.P. Maishev. Silicon Oxycarbide thin films deposited from viniltrimeth oxysilane ion beams. Thin Solid Films, 2005 (492) 114-117.

[12] Z.R. Yan . Development direction of semiconductor wafer cleaning technology. Eqpt. Elec. Ind. 2004, (09) 23-26. 